\title{
Paracontrast and disinhibition
}

\author{
THOMAS C. PRAGER and HALSEY H. MATTESON \\ Tulane University, New Orleans, Louisiana 70118
}

\begin{abstract}
This study examined the possibility that paracontrast results from reduction of mask effectiveness by the test stimulus. The stimuli were two stripes flanking a central rectangle. There was no sign of increased test brightness (disinhibition) and little evidence of paracontrast with the central rectangle as the test stimulus. With the flanking stripes as the test stimulus, disinhibition occurred at test-mask asynchronies corresponding to asynchronies at which the effect of test (stripes) on mask (rectangle) was greatest. Brightness reduction adjacent to disinhibition could be interpreted as paracontrast. Other situations in which effects of test on mask could affect the shape of masking curves were discussed.
\end{abstract}

Metacontrast and paracontrast are special cases of masking in which maximum masking occurs with asynchronous stimuli. Maximum metacontrast with suprathreshold brightness-matching measures occurs when test precedes mask by 50 to $100 \mathrm{msec}$ (Alpern, 1953; Matteson, 1969; Schiller \& Smith, 1966). Paracontrast is a weaker, less reliable effect, in which a small amount of masking occurs when mask precedes test by 20 to 70 msec (Alpern, 1953; Stigler, 1910). In the present study, we examined the possibility that reduction of mask effectiveness by the test could explain paracontrast.

Breitmeyer and Ganz (1976) and Weisstein, Ozog, and Szoc (1975) explain metacontrast in terms of interchannel inhibition. Transient channels respond rapidly to low spatial frequencies, and sustained channels respond slowly to high spatial frequencies. Any task requiring detection of high spatial frequencies is mediated by sustained units, and this includes suprathreshold brightness measures, since brightness depends on the luminance distribution at stimulus edges (Weisstein et al., 1975). If metacontrast results from transient units stimulated by the mask overtaking and inhibiting sustained units stimulated by the test (interchannel inhibition), maximum masking should occur when test precedes mask.

Intrachannel inhibition is a common explanation of paracontrast (Breitmeyer \& Ganz, 1976; Nye \& Naka, 1971). Inhibitory-surround response lags excitatorycenter response by 10 to $30 \mathrm{msec}$ in lateral geniculate cells (Poggio, Baker, Lamarre, \& Sanseverino, 1969; Singer \& Creutzfeldt, 1970) and by $80 \mathrm{msec}$ in retinal ganglion cells (Nye \& Naka, 1971). Since inhibitorysurround response is slower than excitatory-center response, masking due to lateral inhibition in lateral geniculate or retinal ganglion cells should be largest

This paper is based on a thesis submitted by the first author in partial fulfillment of the requirements for the Master of Science degree in psychology at Tulane University. These data were reported at the annual meeting of the Psychonomic Society, Washington, D.C., November 1977. when mask precedes test. Breitmeyer and Ganz (1976) treat Alpern's (1953) metacontrast-paracontrast functions as the superimposition of two separate curves. The large metacontrast effect is due to transient mask units inhibiting sustained units stimulated by the test (interchannel inhibition). A small reduction of test-stimulus effectiveness when mask precedes test (paracontrast) results from intrachannel inhibition. The sum of the two functions is a bimodal function. Weisstein et al. (1975) also take a superimposition approach, but they explain paracontrast in terms of transient units stimulated by the test being inhibited by sustained components of the response to the mask.

An alternative explanation of bimodal metacontrastparacontrast functions is that the test might reduce mask effectiveness. If effects of test on mask are negligible, a strong metacontrast effect would be expected with a brightness-matching measure, but monotonically decreasing forward masking would be expected due to spread of masking effect over time when mask precedes test. If test does affect mask, increased test brightness (disinhibition) should be largest at the test-mask asynchrony at which the effect of test on mask is largest. Paracontrast (brightness reduction) would be found adjacent to disinhibition. This study is based on the assumption that mask brightness is proportional to mask effectiveness. To test this notion of disinhibition, a three-rectangle stimulus configuration was used. Either the center rectangle or the flanking rectangles served as the test, depending on which was attended to. Usually (Alpern, 1953), the flanking rectangles (mask) surround the long sides of the test, so the pair of rectangles is an effective mask. With a split test (flanking rectangles), the single-rectangle mask would be expected to be relatively ineffective, since it does not surround the test stimulus.

With both the center-rectangle test and the split test, minimum test brightness was predicted when test preceded mask (i.e., a metacontrast effect). With the rectangle test, increased test brightness (disinhibition) was predicted at an asynchrony corresponding to the 

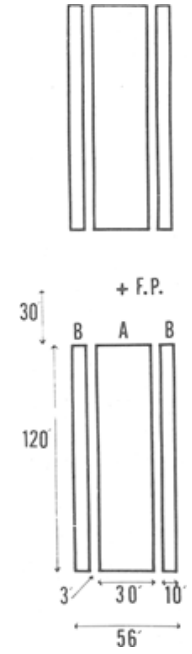

Figure 1. Stimulus configuration.

interval at which the center rectangle (test) had maximum effect on the flanking rectangles (mask). With the split test (flanking rectangles), it was predicted that there would be increased test brightness (disinhibition) at an asynchrony at which stripes (test) had maximum masking effect on the central rectangle (mask). Since the split test is an effective masking stimulus and the rectangle is not, the split test should result in a greater effect of test on mask than the center-rectangle test.

\section{METHOD}

The three-channel Maxwellian view optical system used in this study was described previously (Matteson, 1969). The light source was a ribbon-filament tungsten bulb. Timing was controlled with a digital timer and electromagnetic stepping motors. A biting bar mounted on a calibrated stage was used to maintain head position. Both observers, T.P. and H.M., were males, aged 29 and 39, respectively. Both were myopic and had normal acuity with eyeglasses. Both had a minimum of $30 \mathrm{~h}$ of practice before the experiment.

The stimulus configuration is diagrammed in Figure 1. Test and masking stimuli were presented to the right eye $30 \mathrm{~min}$ above a dim fixation light. Two narrow 10-min stripes flanked a wider 30-min rectangle, with a 3-min separation between stripes and rectangle. The stripes (B) or the rectangle (A) served as test or mask, depending on instructions to the observer. The comparison stimulus was identical to the test-mask combination (stripes and rectangle together) and was presented $30 \mathrm{~min}$ below fixation in the left eye. Duration of all stimuli was $5 \mathrm{msec}$. Test and comparison stimuli were presented simultaneously. Test and mask luminance was constant at 1,800 trolands. White noise in earphones, beginning $1.5 \mathrm{sec}$ prior to trial onset, served both as a ready signal and to mask shutter clicks.

A point of subjective equality (PSE) was obtained by varying comparison luminance to match test brightness. PSEs were obtained with the method of limits, with random starting points and a staggered step size of 30 arbitrary units $(.26 \mathrm{log})$. Under each condition, two ascending and two descending series were presented in random order. Alternating series were a half-step apart $(.13 \mathrm{log})$. For example, if Trial 1 had steps of 10,40 , and 70 , Trial 2 steps would be $25,55,85$, and so on. Intertrial interval was $10 \mathrm{sec}$. The 14 stimulus onset asynchronies (SOAs) were $+300,+200,+100,+75,+50,+25,0,-25,-50,-75$, $-100,-200,-300 \mathrm{msec}$, and a no-mask control. Positive values indicate that mask onset preceded test onset, and negative indicate that test preceded mask. Each session began with 5 min of dark adaptation and four practice series with no mask. After four SOA conditions, a 5-min rest was given. After a 1 -h rest, a second session was conducted, so that a complete presentation of all SOAs with two no-mask controls was given each day. The mean of two determinations was used as the PSE for the nomask control. Each day involved either the center-rectangle test or the split (flanking-stripes) test, but order of presentation was randomized over days. Observers were told which stimulus to judge. Order of presentation of SOA conditions was random. The experimental design was a repeated-measures factorial: test (stripes vs. rectangle) by SOA by replications by subjects, with four replications.

\section{RESULTS}

The largest reductions of test-stimulus brightness occurred consistently at $-75 \mathrm{msec}$ (see Figure 2). The main effect of SOA was statistically significant at the .05 level. Since the interaction of subjects with test was significant at the .05 level, separate test by SOA by replications analyses of variance were conducted for each observer. The test main effect was significant at the .01 level for H.M. Although there was not a significant test main effect for T.P. ( $p>.05)$, there was more masking at $-75 \mathrm{msec}$ with the stripe mask than with the center-rectangle mask. Although both observers showed maximum masking at $-75 \mathrm{msec}$, H.M.'s masking effect was very large with the center-rectangle test. This difference between observers can also account for the subjects main effect $(p<.01)$, the interaction of SOA

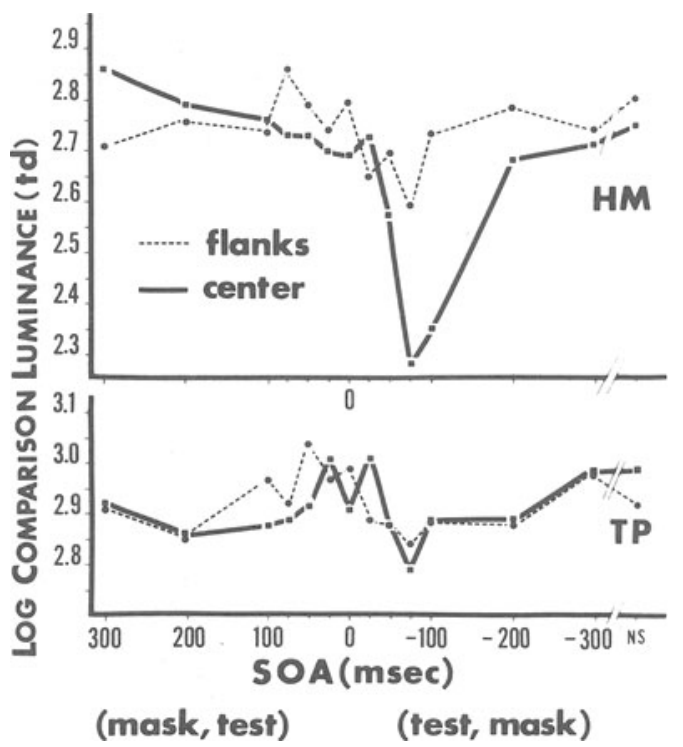

Figure 2. Comparison-stimulus retinal illuminance to match test-stimulus brightness as a function of SOA for observers H.M. (top) and T.P. (bottom). Solid lines denote the central test condition (rectangle test, stripes as mask), and dotted lines indicate the split-test condition (flanking stripes as test, rectangle mask). Control measures for each condition (labeled NS) indicate mean PSE obtained with no masking stimulus. 
with test $(p<.01)$, and the interaction of test with subjects $(\mathrm{p}<.05)$.

\section{DISCUSSION}

As is typical, metacontrast was much stronger than paracontrast. Masking was greatest at $-75 \mathrm{msec}$ for both observers with the split (flanking-stripes) test as well as with the centerrectangle test. Although the effect was much larger for H.M., both observers showed more metacontrast with the rectangle test than with the split test. This was expected, since the rectangle test was more completely surrounded by its mask than was the split test. A test that is more completely surrounded by the inducing field results in more contrast than a test that is partially surrounded by the inducing field with extendedduration stimuli in the standard contrast paradigm (Horeman, 1963). Since metacontrast decreases with increased spatial separation between test and mask (Alpern, 1953; Growney, Weisstein, \& Cox, 1977), the split test, which has its outer edges further removed from the mask than the center-rectangle test, should be masked to a lesser degree than the central rectangle.

There was no evidence of disinhibition with the centerrectangle test. There was no sign of increased brightness near +75 msec (mask preceding test) on the rectangle test curves, which would correspond to the maximum effect of the rectangle (test) on the stripes (mask). Since the rectangle was an ineffective mask, the absence of disinhibition with the rectangle test is not surprising. Using Breitmeyer and Ganz's (1976) standard of 10 to $30 \mathrm{msec}$, there is no sign of paracontrast; however, T.P. did show brightness reduction at long positive intervals $(+100$ and +200$)$, and this reduction of test brightness could be called paracontrast. With the usual three-rectangle configuration with the central rectangle as the test stimulus, effects of test on mask are probably negligible. Small effects of test on mask may be the reason that three-rectangle and diskring configurations with the test stimulus in the middle are the standard practice.

There was evidence of disinhibition with the split test. Both observers showed increased comparison luminance near +75 msec [mask (rectangle) preceding test], which corresponds to the SOA at which effects of stripes on rectangle were greatest ( $-75 \mathrm{msec}$ on the rectangle test curve). H.M.'s maximum test brightness occurred at $+75 \mathrm{msec}$, and T.P.'s maximum was at +50 , with some increased brightness at +75 and +100 msec. The split test, which is an effective masking stimulus, inhibited the center-rectangle mask. This study indicates that disinhibition resulting from masking of the mask by the test stimulus can be a factor when the test stimulus is an effective mask.

There are other situations in which effects of test on mask could be a stronger effect than it was in the present study. Matin (1975) argued that with the three-rectangle configuration, spatial asymmetry results in different stimulation when mask precedes test than when test precedes mask. Matin suggested that spatial asymmetry could be eliminated by presenting two identical rectangles, one as test and one as mask. Since the test stimulus would result in just as much masking as the masking stimulus, disinhibition could be appreciable. In the present study, although masking of the flanking stripes by the central rectangle was a small effect, maximum masking occurred at the same SOA as with the center-rectangle test. Thus, it would seem that spatial asymmetry is not a crucial factor in determining the SOA at which maximum masking occurs.

Disinhibition could occur with monotonic masking functions (i.e., maximum masking when test and mask are simultaneous). If the effects of test on mask are also maximum with simultaneous presentation of test and mask, it is possible that effects of test on mask could reduce mask effectiveness near test-mask synchrony, thereby converting a monotonic masking function

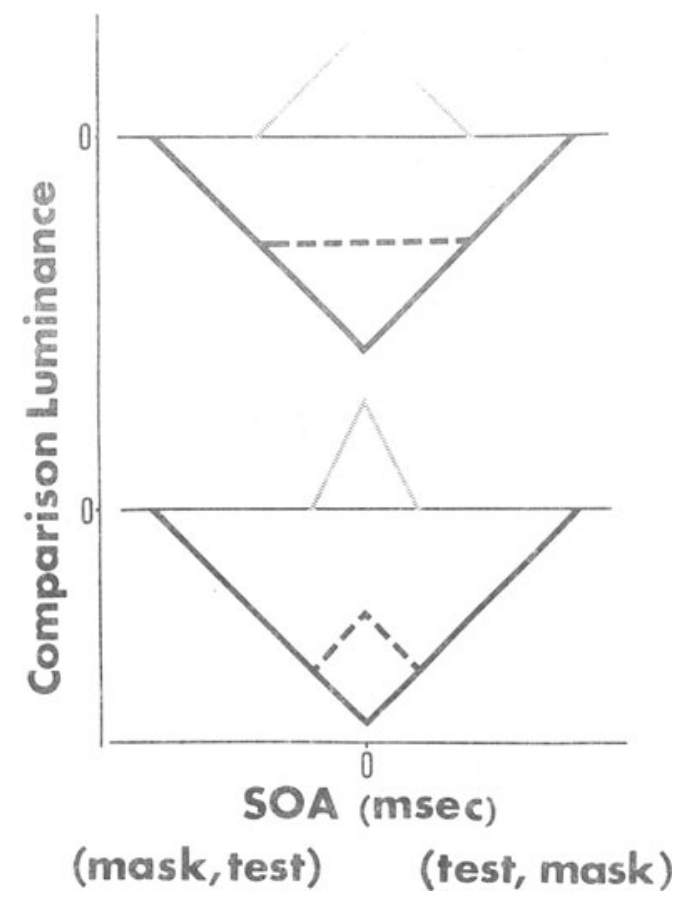

Figure 3. Hypothetical masking curves showing how reduction of masking-stimulus effectiveness by the test stimulus could affect the shape of the resultant masking curve. The horizontal axis in each inset (0 on the vertical axis) represents comparison luminance with no mask presented. The resultant masking curve was obtained by subtracting effects of test on mask (shaded line) from effects of mask on test (heavy solid line). The resultant masking curve is indicated by the broken line where it differs from the curve showing effects of mask on test.

into a W-shaped curve (Figure 3, lower half). One limitation of this approach is that the masking curve showing effects of test on mask would need to have a steeper slope than the curve showing effects of mask on test; otherwise, effects of test on mask would only flatten the bottom of the masking curve (Figure 3, upper half). Even with this limitation in mind, Saunders' (1977) metacontrast effect might be explained in terms of effects of test on mask. Using a three-rectangle configuration, Saunders varied mask luminance to match test brightness. The mask served both as mask and comparison stimulus. A steeper function for effects of test on mask is a possibility in this case, because masking of mask by test would attenuate both mask effectiveness and comparison effectiveness. Saunders had no SOAs at which mask preceded test; therefore, it is possible that his metacontrast curves are actually half of a W-shaped function. Stoper and Mansfield (Note 1) presented W-shaped masking curves. Since their test stimulus was a 5-deg circle, and the mask was two 1-deg squares spaced $1 \mathrm{deg}$ apart, the small mask could result in disinhibition due to large effects of test on mask with a maximum at test-mask synchrony.

In order for disinhibition to occur, there must be appreciable masking at the SOA at which effects of test on mask are greatest. In other words, disinhibition is masking of the masking stimulus; therefore, disinhibition will not be appreciable unless the masking stimulus is doing some masking in the first place. It is likely that the disinhibition effect obtained in the present study with the split-test stimulus was small because there was little masking when mask preceded test by $75 \mathrm{msec}$. Studies showing disinhibition with additional masking stimuli adjacent to the mask (Dember \& Purcell, 1967; Robinson, 1966, 1968) 
were obtained with detection measures, and maximum masking occurred when test and mask were simultaneous (monotonic functions). The present study also depends on the assumption that mask effectiveness is proportional to mask brightness, an assumption that is not always reasonable (Cornsweet \& Teller, 1965). Unless there are good a priori reasons to discount the possibility of appreciable effects of test on mask, effects of test on mask should be measured.

\section{REFERENCE NOTE}

1. Stoper, A. E., \& Mansfield, G. Metacontrast and paracontrast of a contourless area. Paper presented at the annual meeting of the Psychonomic Society, Washington, D.C., November 1977.

\section{REFERENCES}

AlPERn, M. Metacontrast. Journal of the Optical Society of America, 1953, 43, 648-657.

BREITMEYER, B. G., \& GANz, L. Implications of sustained and transient channels for theories of visual pattern masking, saccadic suppression, and information processing. Psychological Review, 1976, 83, 1-36.

Cornsweet, T. N., \& Teller, D. Y. Relation of increment thresholds to brightness and luminance. Journal of the Optical Society of America, 1965, 55, 1303-1308.

Dember, W. N., \& Purcell, D. G. Recovery of masked visual targets by inhibition of the masking stimulus. Science, 1967, 157, 1335-1336.

Growney, R., Weisstein, N., \& Cox, S. I. Metacontrast as a function of spatial separation with narrow line targets and masks. Vision Research, 1977, 17, 1205-1210.

HoREMAN, H. W. Inductive brightness depression as influenced by configurational conditions. Vision Research, 1963, 3, 121-135.
Matin, E. The two-transient (masking) paradigm. Psychological Review, 1975, 82, 451-461.

Matteson, H. H. Effects of surround size and luminance on metacontrast. Journal of the Optical Society of America. 1969, 59, 1461-1468.

NyE, P., \& NAKA, K. -I. The dynamics of inhibitory interaction in a frog receptive field: A paradigm of paracontrast. Vision Research, 1971, 11, 377-392.

Poggio, G. B., Baker, F. H., Lamarre, Y., \& Sanseverino, E. R. Afferent inhibition at input to visual cortex of the cat. Journal of Neurophysiology, 1969, 32, 916-929.

Robinson, D. N. Visual disinhibition of visually masked stimuli. Science, 1966, 154, 157-258.

Robinson, D. N. Visual disinhibition with binocular and interocular presentations. Journal of the Optical Society of America, 1968, 58, 254-257.

SAUnders, J. E. Foveal and spatial properties of brightness metacontrast. Vision Research, 1977, 17, 375-378.

Schiller, P. H., \& SMITH, M. C. Detection in metacontrast. Journal of Experimental Psychology, 1966, 71, 32-39.

Singer, W., \& CREUTzFELdT, O. D. Reciprocal lateral inhibition of on- and off-center neurones in the lateral geniculate body of the cat. Experimental Brain Research, 1970, 10, 311-330.

STIGLER, R. Chronoptische Studien über den Umgebungskontrast. Pflügers Archiv Gesamte Physiologie der Menschen und Tiere, 1910, 134, 365-435.

Weisstein, N., Ozog, G., \& Szoc, R. A comparison and elaboration of two models of metacontrast. Psychological Review, 1975, 82, 325-343.

(Received for publication July $31,1978$. 